\title{
A Review on Content based Image Retrieval
}

\author{
Tanvi Patel \\ PG student Department Of \\ Electronics And \\ Communication Engineering, \\ Uka Tarsadia University, \\ Guajart
}

\author{
Mayank Kapadia \\ Department Of Electronics And \\ Communication Engineering, \\ Uka Tarsadia University, \\ Guajart
}

\author{
Jemish Maisuria \\ Department Of Electronics And \\ Communication Engineering, \\ Uka Tarsadia University, \\ Guajart
}

\begin{abstract}
Literature survey is an important for understanding and gaining much more knowledge about the specific area of a subject. An image retrieval system is a computer system for browsing, searching and retrieving images from a large database of digital images. Content-based image retrieval (CBIR) is an image search technique that complements the traditional text-based retrieval of images by using visual features, such as color, texture, and shape. This system retrieve according to the query image; that is, the user provides or selects a query image and chooses a distance measure that will be used to compare the query image to the images stored in the database. This paper is attempt to explore different CBIR technique and their application.
\end{abstract}

\section{General Terms}

Content based image retrieval(CBIR), color, shape, texture, feature vector, classification, principle of CBIR, performance parameter

\section{Keywords}

CBIR(Content based image retrieval) system, colour histogram, GLCM, edge histogram, feature vector

\section{INTRODUCTION}

During the past years, Content Based Image Retrieval (CBIR) has gained much for its potential application in multimedia management. In the current stage, an effective search engine is needed for huge database. "Content-based" means that the search analyses, the contents of the image rather than the metadata such as keywords, descriptions or tags associated with the image. The term "content" might be referred as color, shape, texture, or any other information that can be derived from the image itself. It is also known as Query By Image Content (QBIC) or Content-Based Visual Information Retrieval (CBVIR). The CBIR system have used in varies applications such as Medical diagnosis, Crime prevention, Fashion and interior design, Architectural and engineering, Journalism and advertising, Education and training, Cultural heritage, Home entertainment, Geographical information and remote sensing system and web searching.

Early techniques were not based on visual features but on the textual annotation. The images were annotated by text and then searched using text based approach. In General, text based image retrieval system is inefficient. For the huge amount of image data, vast amount of labour required in manual annotation. For every visual feature description within image is difficult and time consuming. So instead of manual annotation by text based descriptions or keywords, images are described by visual features like shape, texture, color.

In CBIR no additional information such as text annotations, place of creation or time is available. The retrieval problem is solved by content of image based on available characteristics of pixels. An alternative method of CBIR is description based image retrieval (DBIR). For DBIR, retrieval is done if all images of dataset have annotations describing their content. A general CBIR system use different type of queries like provides relevant images from given database, query by example, sketch or region, based not on media metadata textual annotation but on similarity function using low level features.

\section{FEATURE EXTRACTION}

\subsection{Color feature}

Color feature is the most significant in searching desired image from database. Color plays important role in the human visual perception system. Different methods for representing color feature are divided into two groups: color histogram and statistical method of color representation [1]. Color is represented by histogram. Color histogram is invariant to scaling, rotation and translation of the object [1].

\subsection{Texture feature}

Texture gives the information on structural arrangement of objects and surfaces of the images. Texture is not defined for a separate pixel but it depends on the distribution of intensity of the image [1]. Texture possesses scalability and periodicity properties; it can be described by main directions, sharpness and contrast. Texture plays an important role in comparison of images supplementing colour feature. It contains important information related to the structural arrangement of the surface, such as leaves, fabric, clouds and bricks [3][7].

\subsection{Shape feature}

Along with texture and color feature, shape of objects is also used for image comparison [1]. Here shape is not referred as shape of whole image but consider as shape of each object within the image. Methods for describing and representing shapes can be divided into two groups: Internal methods, which represent the region in terms of its internal characteristics i.e. the pixels comprising the region. External methods, which represent the region in term of external characteristics i.e. its boundary. Shape is invariant to translations, rotations, and scaling [1].

\section{FEATURE EXTRACTION METHOD}

\subsection{Color feature extraction}

Several methods for retrieving images on the basis of color similarity have been described in the literature, but most of variations on the same basic idea. Each image added to the database to analyzed color histogram, which shows the proportion of pixels of every color within the image. The color histogram for each image is stored in the vector within database. At search time, the user can either specify the desired proportion of each color or submit an image whose color histogram is calculated. Other way, the matching process retrieves those images whose color histograms match the query image closely. The matching technique commonly 
used, histogram intersection, was first developed. Variants of this technique is now used in a high proportion of current CBIR systems.

\subsubsection{RGB color model}

The RGB color model is composed of the primary colors Red, Green, and Blue. This system defines the color model that is used in most color CRT monitors and color raster graphics. They are considered as the "additive primaries" since the colors are added together to produce the desired color. The RGB model use the Cartesian coordinate system. Notice the diagonal from $(0,0,0)$ black to $(1,1,1)$ white which represents the grey-scale. Figure 1 shows the view of the RGB Color Model looking down from "White" to origin.

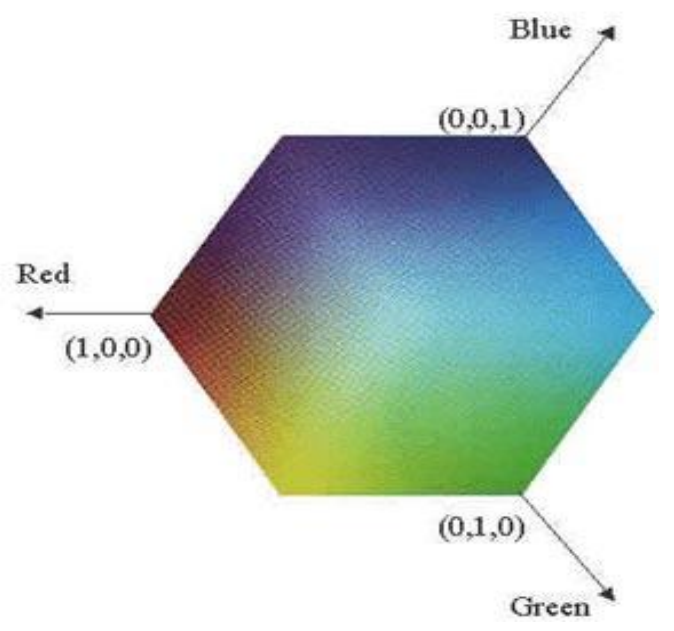

Fig 1. RGB color model [14]

\subsubsection{HSV color model}

The HSV stands for the Hue, Saturation and Value. The coordinate system is a hexagon. The Value represents intensity of a Color, which is decoupled from the color information in the represented image. The saturation and hue components are related to the way of human eye perceives color resulting in image processing algorithm.

\subsubsection{Hие}

Hue represents the dominant wavelength in light. Hue is the term for the spectrum colors. Hue is expressed from $0^{\circ}$ to $360^{\circ}$. It can represent hues of red starts with $0^{\circ}$, yellow starts with $60^{\circ}$, green starts with $120^{\circ}$, cyan starts with $180^{\circ}$, blue starts with $240^{\circ}$ and magenta starts with $300^{\circ}$.

\subsubsection{Saturation}

Saturation represents the dominance of hue in color. It can also be thought as the intensity of the color.

\subsubsection{Value}

It can describe the intensity or brightness of the color. In other words value is defined as a relative darkness or lightness of color.

\subsubsection{Conversion of RGB to HSV:}

\section{BLUE}

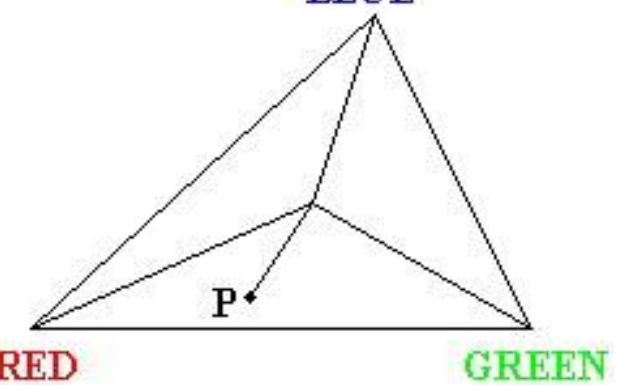

Fig 2. RGB to HSV conversion [14]

$$
H=\operatorname{Cos}^{-1} \frac{1 / 2[(R-G)+(R-G)]}{\sqrt{(R-G)^{2}+(R-B)(R-G)}}
$$

$$
S=1-\frac{3[\min R G B]}{(R+G+B)}
$$

$$
V=\frac{(R+G+B)}{3}
$$

\subsubsection{Histogram}

Histogram can be used for color feature extraction. Color histogram can be representing as a distribution of colors in image. Histogram can convert image in one domain to another i.e. RGB color space to other space[14]. In General convert RGB color space to HSV space. They can calculate by below steps:

Step 1: convert RGB space to HSV space.

Step 2: quantified the image.

Step 3: calculate the feature value.

\subsection{Texture feature extraction}

Texture is important characteristics of a material defining the appearance of it's surface. Different approaches for texture characterization are based on DWT(wavelet transform)[3], GLCM(Grey-Level Co-occurrence Matrix)[7] and Gabor filter [7]. In this paper, texture is analyzed through second order statistical measurement based on the Grey-Level Cooccurrence Matrix proposed by Haralick.

\subsubsection{GLCM(Gray Level Co-occurrence Matrices):}

A statistical method of examining texture that considers the spatial relationship of pixels is the gray-level co-occurrence matrix (GLCM), also known as the gray-level spatial dependence matrix [11]. The GLCM functions characterize the texture of an image by calculating how many pairs of pixel with specific values and in a specified spatial relationship occur in an image. 


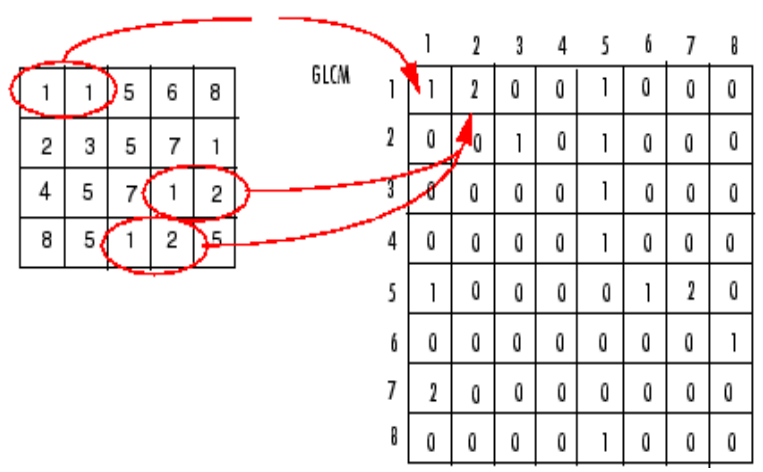

Fig 3. Conversion of image to GLCM [11]

\subsubsection{Contrast:}

Contrast is a local gray level variation in the GLCM. It can be thought of as a linear dependency of gray levels of neighboring pixels. Contrast is 0 for constant image [2][7].

$$
\text { Contrast }=\sum|i-j|^{2} p(i, j) \text {. }
$$

Where $p(i, j)=$ Probability Density

\subsubsection{Homogeneity:}

Homogeneity measures the uniformity of the non-zero entries in the GLCM. It weights values by the inverse of contrast weight [2][7].

$$
\text { Homogeneity }=\frac{\sum \mathrm{p}(\mathrm{i}, \mathrm{j})}{1+|\mathrm{i}-\mathrm{j}|}
$$

\subsubsection{Energy:}

Energy is a measure of local homogeneity and therefore it represents the opposite of the Entropy. Basically this feature will tell us how uniform the texture is [2][7],

Energy $=\sum p(i, j)^{2}$

\subsubsection{Entropy:}

Entropy in any system represents disorder, where in the case of texture analysis is a measure of its spatial disorder [2][7].

Entropy $=\sum p(i, j) \log (p(i, j))$

\subsection{Shape feature extraction}

For a shape feature extraction edge histogram method can be used. The edges give an idea about the shapes of objects present in the image .So they are useful for registration, identification and segmentation of objects in a image.

Edge is basic feature of image that contain contour information of object in image. So, edge feature cannot express only the content of image but it is use for object recognition. Although the canny, prewitt and sobel operators can separate the edge information from the background but the generally the edge image is complicated and cannot constitute contours and it contain huge number of lines and curves that are difficult to described by mathematical formula [2].

In this paper, extended edge histogram method, combined with GLCM. Firstly, texture feature are extracted using
GLCM and than shape feature are extracted and edge histogram was computed.

Edge histogram is a visual description for shape feature of image. Based on edge histogram, six types of edges for describe information of contour for every sub-region. They are empty, horizontal, vertical, $45^{\circ}$ direction, $135^{\circ}$ direction and chaos as shown fig 6 [2].
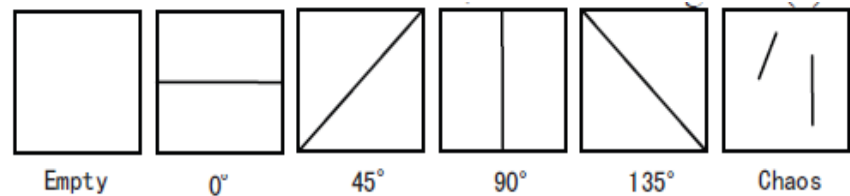

(a)
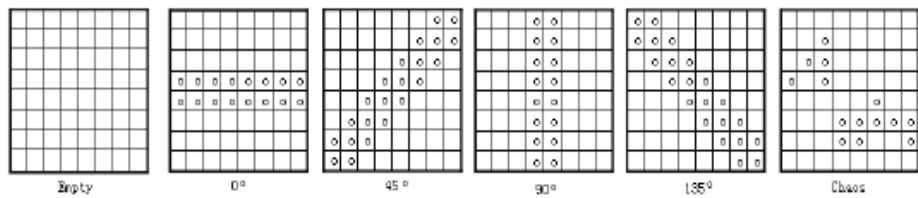

Fig 4. Edge type and discriminance: (a) Edge type (b) corresponding discriminance [2]

Steps for this discriminance below [2]:

1. Establish four Boolean matrix $\mathbf{M}_{0}, \mathrm{M}_{90}, \mathbf{M}_{45}$ and $\mathrm{M}_{135}$ corresponding edges "Horizontal", "Vertical", " $45^{\circ}$ direction" and " $135^{\circ}$ direction".

2. Compute the total number of edge pixels, denoted by totalpix.

3. If totalpix $\leq \mathrm{T}$, the sub-block is "Empty" type.

4. Compute edge histogram by following formula:

$$
\mathrm{H}_{\text {sub }}(\mathrm{k})=\frac{\sum \sum \mathrm{E}(\mathrm{i}, \mathrm{j})}{\text { Nall }} \ldots
$$

Where $E(i, j)=$ edge type

\section{SIMILARITY AND PERFORMANCE MEASUREMENT PARAMETER}

\subsection{Similarity Feature Extraction}

The similarity feature which is used for comparing the various features. For retrieve the Similarity images from the large image database, three types of measurements are used for similarity extraction[14].

\subsubsection{Euclidean Distance}

Euclidean distance is defined as the displacement of a pixel from the nearest background point. The equation of Euclidean distance is[14]

$$
\mathrm{D}=\sum(\mathrm{Ai}-\mathrm{Bi}) 2
$$

\subsubsection{Chi Square Distance}

The Euclidean distance between the components of profiles, on which weighting is defined (weight means the inverse of its frequency), is called the chi-square distance. The equation of Chi-square distance is[14]

$\chi_{\text {th }}^{2}=\sqrt{\sum_{j=1}^{p} \frac{1}{a_{+j}}\left[\frac{a_{h j}}{a_{h+}}-\frac{a_{i j}}{a_{i+}}\right]^{2} \ldots}$ 


\subsubsection{Weighted Euclidean Distance}

Multiply squared differences by corresponding weights are known as Weighted Euclidean Distance. The formula of Weighted Euclidean Distance is[14]

$\mathrm{dx}, \mathrm{y}=\sqrt{\sum_{\mathrm{j}=1}^{\mathrm{J}} \frac{1}{\mathrm{~S}_{\mathrm{j}}^{2}}}\left(\mathrm{x}_{\mathrm{j}}-\mathrm{y}_{\mathrm{j}}\right) 2$

\subsection{Performance Parameter}

Evaluation of retrieval process is a crucial problem in CBIR. Different methods are used for measuring performance of retrieval system. The most common performance parameters are Precision and Recall .

\subsubsection{Precision}

Precision rate is defined as a ratio of number of retrieve relevant images similar to the query to the total number of retrieved images in response to query[1][10[11].

Precision $==$ Number of relevant images retrieved

Total number of images retrieved

\subsubsection{Recall}

Recall rate is defined as a ratio of number of retrieve relevant images similar to the query to the total number of relevant images available in the database[1][10][11].

Recall $==$ Number of relevant images retrieved

Total number of images in database

\section{CONCLUSION}

This paper has review of essential concepts of content based image retrieval. Use of visual feature such as texture, color and shape feature vector to match the image can give better region. In this paper, the color features can be extracted using histogram, texture features can be extracted using GLCM algorithm and shape features can be extracted using edge histogram. For similarity measurements Euclidean distance, weighted Euclidean distance and chi-square distance can be used. For a performance measurement precision and recall rate can be used.

Although content-based retrieval provides an automatic and intelligent solution for efficient searching of images, the majority of current techniques are based on low level features or current techniques are primarily based on low level features. The similarity measures between visual features do not necessarily match human perception but user demands it to be in semantically and perceptually similar images, the retrieval results of low level feature based retrieval approaches are generally unsatisfactory and often unpredictable. To solve this problem further research is required.

It is understood that human's perception in view of any image similarity would definitely be semantic, subjective and taskdependent. Content-based methods give some definite directions for image retrieval. But in general results is based on the similarities of pure visual features, does not always be perceptually and semantically meaningful.

CBIR systems are developed to benefit medical field by the means of clinical research and also educational features of biomedicine those are designed for many classes for biomedical images. And it is essential for a system to grow suitable features and required a similarity algorithm to capture the contents efficiently in an image.

\section{REFERENCES}

[1] Yogita Mistry 1, Dr.D.T. Ingole, 'Survey on Content Based Image Retrieval Systems', IJIRCCE International Journal of Innovative Research in Computer and Communication Engineering, Vol. 1, October 2013.

[2] Peiqiang Zhang, Hongguang Zhu, 'Medical Image Retrieval Based on Co-Occurrence Martix and Edge Histogram', IEEE 2010.

[3] S. Arivazhagan, L. Ganesan b, 'Texture Segmentation Using Wavelet Transform', Pattern Recognition Letters 24 (2003) 3197-3203.

[4] Hassan Farsi, Sajad Mohamadzadeh, 'Colour And Texture Feature-based Image Retrieval By Using Hadamard Matrix In Discrete Wavelet Transform', IET Image Process., 2013, Vol. 7, Iss. 3, pp. 212-218.

[5] James Z. Wang, Jia Li, and Gio Wiederhold, 'SIMPLIcity: Semantics-Sensitive Integrated Matching for Picture Libraries', IEEE Transactions On Pattern Analysis And Machine Intelligence, VOL. 23, NO. 9, September 2001.

[6] Haoyu Ren, Ze-Nian $\mathrm{Li}$, 'Object Detection Using Edge Histogram Of Oriented Gradient', IEEE 2014.

[7] A. Gebejes, R. Huertas, 'Texture Characterization based on Grey-Level Co-occurrence Matrix', Conference of Informatics and Management Sciences March, 25. - 29. 2013.

[8] A.Srinagesh, K.Aravinda, G.P.Saradhi Varma, A.Govardhan, M. SreeLatha, 'A Modified Shape Feature Extraction Technique For Image Retrieval', International Journal of Emerging Science and Engineering (IJESE) ISSN: 2319-6378, Volume-1, Issue-8, June 2013.

[9] Jun Yue, Zhenbo Li, Lu Liu and Zetian Fu, "Content Based Image Retrieval Using Color And Texture Fused Features”, ELSEVIER ,pg.no. 1121-1127,2011.

[10] Suman Lata and Parul Preet Singh, 'A Review on Content Based Image Retrieval System', International Journal of Advanced Research in Computer Science and Software Engineering (IJARCSSE), ISSN: 2277 128X, Volume 4, Issue 5, May 2014.

[11] Amanbir Sandhu and Aarti Kochhar, 'Content Based Image Retrieval Using Texture, Color And Shape For Image Analysis', International Journal of Computers \& Technology (IJCT),Volume 3, No. 1, AUG, 2012.

[12] Shankar M. Patil, 'Content Based Image Retrieval Using Color, Texture \& Shape', International Journal of Computer Science \& Engineering Technology (IJCSET), ISSN: 2229-3345, Vol. 3 No. 9 Sep 2012.

[13] P. Mohanaiah, P. Sathyanarayana, L. GuruKumar, 'Image Texture Feature Extraction Using GLCM Approach', International Journal of Scientific and Research Publications, Volume 3, Issue 5, May 20131 ISSN 2250-3153.

[14] Ms. K. Arthi and Mr. J. Vijayaraghavan , 'Content Based Image Retrieval Algorithm Using Colour Models', International Journal of Advanced Research in Computer and Communication Engineering ((IJARCCE ), Vol. 2, Issue 3, March 2013. 